Research Paper

\title{
Over-expression of Activated Signal Transducer and Activator of Transcription 3 Predicts Poor Prognosis in Upper Tract Urothelial Carcinoma
} \author{
$1,2,3 \bowtie$ \\ 1. Department of Urology, Kaohsiung Medical University Hospital, Kaohsiung Medical University, Kaohsiung, Taiwan \\ 2. Graduate Institute of Medicine, College of Medicine, Kaohsiung Medical University, Kaohsiung, Taiwan \\ 3. Department of Urology, School of Medicine, College of Medicine, Kaohsiung Medical University, Kaohsiung, Taiwan \\ 4. Department of Urology, Ministry of Health and Welfare Pingtung Hospital, Pingtung, Taiwan \\ 5. Center for Infectious Disease and Cancer Research, Kaohsiung Medical University, Kaohsiung, Taiwan \\ Department of Anatomy, School of Medicine, College of Medicine, Kaohsiung Medical University, Kaohsiung, Taiwan \\ 7. Center for Stem Cell Research, Kaohsiung Medical University, Kaohsiung, Taiwan \\ 8. Department of Urology, Kaohsiung Municipal Ta-Tung Hospital, Kaohsiung, Taiwan \\ 9. Department of Microbiology, Kaohsiung Medical University, Kaohsiung, Taiwan
}

Wei-Ming Li 1,2,3,4, Chun-Nung Huang 1,2,3,5, Yi-Chen Lee 2,6 , Szu-Han Chen 1,2, Hui-Hui Lin 1,3, Wen-Jeng Wu 1,2,3,5,7,8, Ching-Chia Li 1,2,3,8, Hsin-Chih Yeh 1,2,3,5,8, Lin-Li Chang 2,9, Wei-Chi Hsu 1,2,3, Hung-Lung Ke

$\square$ Corresponding author: Hung-Lung Ke, Department of Urology, School of Medicine, College of Medicine, Kaohsiung Medical University, No. 100, TzYou 1st Road, Kaohsiung City 807, Taiwan; Tel.: +886-7-3208212; Fax: +886-7-3211033; E-mail address: hunglungke@gmail.com.

(c) Ivyspring International Publisher. This is an open access article distributed under the terms of the Creative Commons Attribution (CC BY-NC) license (https://creativecommons.org/licenses/by-nc/4.0/). See http://ivyspring.com/terms for full terms and conditions.

Received: 2016.08.27; Accepted: 2017.09.12; Published: 2017.10.15

\begin{abstract}
Background: Signal transducer and activator of transcription proteins (STATs) play important roles in gene regulation, cell proliferation, and cell differentiation. We aimed to establish the relationship between phosphorylated STAT3 ( $\mathrm{p}-\mathrm{Ser}$-STAT3) expression and the prognosis of upper tract urothelial carcinoma (UTUC).

Methods: This study retrospectively reviewed 100 patients with pathologically confirmed UTUC at Kaohsiung Medical University Hospital. We quantified the expression of p-Ser-STAT3 in cancer cells by immunohistochemistry, and determined the clinicopathological significance of p-Ser-STAT3 expression and prognostic outcomes in patients with UTUC.

Results: High p-Ser-STAT3 expression was detected in $52 \%$ of UTUC patients. High p-Ser-STAT3 expression was associated with poor recurrence-free survival $(p=0.018)$ and overall survival $(p=$ 0.026). In advanced cancer samples (stage T3/T4), p-Ser-STAT3 expression is the only independent prognostic factor for recurrence-free survival (hazard ratio $=5.91, p=0.01$ ) and cancer-specific survival (hazard ratio $=8.83, p=0.039$ ).
\end{abstract}

Conclusions: The expression of p-Ser-STAT3 can be a potential prognostic marker for cancer recurrence and survival in UTUC, especially in advanced stage cases.

Key words: Upper tract urothelial carcinoma; signal transducer and activator of transcription 3; immunohistochemistry; prognosis

\section{Introduction}

In Western countries, renal pelvic urothelial carcinoma accounts for only $5 \%$ of all renal tumors, and upper tract urothelial carcinoma (UTUC) accounts for $5 \%-10 \%$ of all urinary tract cancers [1]. However, there is an unusually high incidence of UTUC in Taiwan [2], suggesting that there may be specific genetic or environmental factors for UTUC carcinogenesis in the Taiwanese population. There are many studies about the mechanism of bladder cancer carcinogenesis, but few studies regarding UTUC have been conducted. Green et al. called bladder cancer and UTUC "the disparate twins", owing to the many 
different characteristics between the two, including gender distribution, prognosis, tumor location, inherent staging, and intra-cavitary therapy [3]. We have previously proposed several molecules such as cyclooxygenease-2 (COX2) [4], osteopontin (OPN) [5], hypoxia-induced factor 1a (HIF-1a) [6], glutathione S-transferase (GST) [7], and nuclear factor-kB (NFkB) [8] as prognostic biomarkers associated with UTUC. However, accurate prognosis prediction of UTUC is still difficult.

Signal transducer and activator of transcription 3 (STAT3) is an important signaling molecule for many cytokines and growth factor receptors, and is required for murine fetal development. The C-terminal transactivation domain of STAT3 plays an important role in its activation through a tyrosine residue at position 705 and a serine residue at position 727 [9]. Published studies have shown that STAT3 is constitutively activated in many human tumors and induces oncogenesis and anti-apoptosis [10]. In normal cells, ligand-dependent activation of STATs is a transient process, lasting from a few minutes to several hours. However, in tumor cells, STAT proteins remain persistently phosphorylated and consequently remain activated. Phosphorylated STAT3 (pSTAT3) dimerizes and moves to the nucleus, regulating the transcription of target genes. STAT3 target genes include survivin, vascular endothelial growth factor (VEGF), matrix metalloproteinases (MMPs), and E-cadherin; these genes regulate cell proliferation, survival, angiogenesis, metastasis, immune evasion, inflammation, and drug resistance in a tumor microenvironment [11, 12].

Recent studies have shown that overexpression of pSTAT3 significantly correlates with a variety of human cancers, including breast cancer [13], liver [14], and head and neck cancer [15]. To our knowledge, there is only one study about STAT3 expression in urothelial carcinoma, including bladder cancer and UTUC [16]. Since bladder cancer and UTUC share the same histology but different clinical characterisics. The purpose of this study was to evaluate the association between pSTAT3 expression and the clinicopathological characteristics of UTUC.

\section{Patients and Methods}

\section{Surgical specimens and clinicopathological data}

One hundred formalin-fixed UTUC samples were obtained from the Department of Urology, Kaohsiung Medical University Hospital from 1997-2006. All samples were histologically confirmed as transitional cell carcinoma. All the patients received nephroureterectomy and excision of bladder cuff. The data were retracted from medical records retrospectively. Follow-Up protocol was decided according to NCCN guideline. Patients received cystoscopy by 3-month interval within 2 years after surgery and then increasing intervals thereafter. Median follow-up time was 40.39 months and the range between 1 to 136 months. Bladder recurrence was defined as UC proved pathologically. Recurrence-free survival was defined as the time from the date of surgery to the date of bladder recurrence. Cancer-specific survival was calculated from the date of surgery to the date of cancer death. The pathologic grade was classified according to World Health Organization (WHO) histologic criteria, and tumor staging was determined according to the International Union Against Cancer tumor-node-metastasis classification. The clinicopathological parameters were obtained by retrospectively reviewing medical records. The informed consent was provided to the patient and signed before surgery. The tumor specimens were collected from surgical specimen. The study protocol was reviewed and approved by the Institutional Review Board of Kaohsiung Medical University Hospital (KMUH-IRB-20120120).

\section{Immunohistochemical Staining of phosphorylated STAT3 (p-Ser-STAT3)}

Four-micrometer-thick sections

from paraffin-embedded blocks were cut onto precoated slides, followed by deparaffinization, rehydration, and antigen retrieval. Endogenous peroxidase was blocked in accordance with the manufacturer's protocol. The slides were incubated with anti-phospho-STAT3 monoclonal antibody (Ser727, sc-135649, Santa Cruz Biotechnology) at a 1:400 dilution at $4^{\circ} \mathrm{C}$ for overnight. Primary antibodies were detected using the DAKO ChemMateEnVision Kit (K5001; Dako, Carpinteria, CA). Finally, the slides were counterstained with hematoxylin and examined by light microscopy. Notably, only the staining in tumor cells (approximately 1000 cells in 3-4 high-power fields) was calculated.

\section{Evaluation of immunohistochemistry staining}

Breast carcinoma samples served as positive controls owing to their constitutive p-Ser-STAT3 activation, according to the producer's suggestions. Sections incubated with no primary antibody were used as negative controls. For each slide, the nuclear immunoreaction in tumor cells was scored separately by two pathologists. The evaluation of p-Ser-STAT3 staining was based on the percentage of positively stained cells in two categories: low expression, $\leq 30 \%$ positive cells; high expression $>30 \%$ positive cells. 

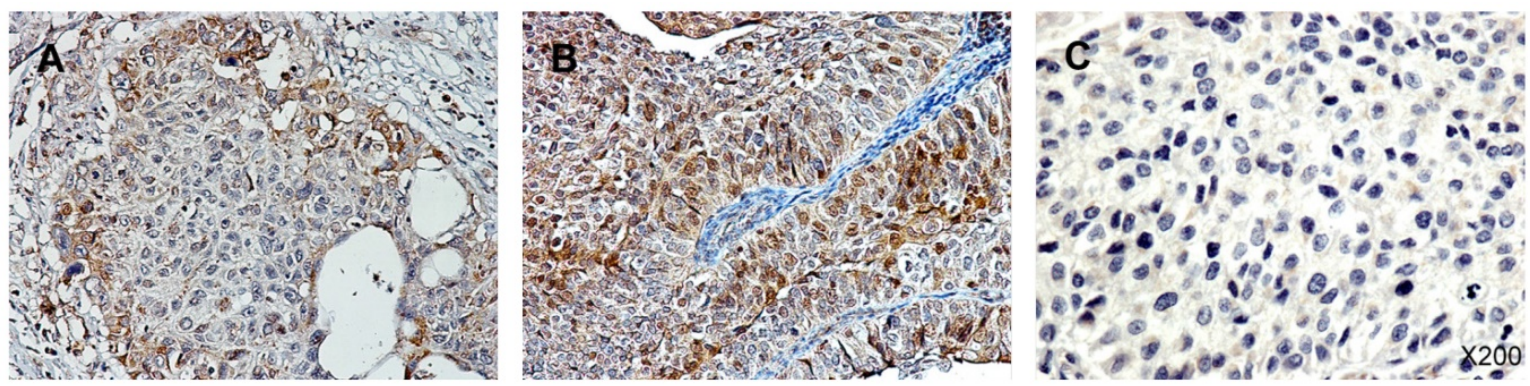

Figure 1. Immunohistochemistry staining for p-Ser-STAT3 in upper tract urothelial carcinoma (UTUC). (A) Low p-Ser-STAT3; (B) High p-Ser-STAT3; (C) negative control.

\section{Statistical Analysis}

Chi-square analysis or Fisher's exact test were used to evaluate p-Ser-STAT3 expression in patients with different age (dichotomized by medium), gender, tumor stage and grade (low or high), creatinine level, and hemodialysis. Hazard ratios (HRs) and 95\% confidence intervals (CIs) computed from univariate and multivariate Cox regression models were used to investigate the relationship between clinicopathological characteristics and survival. Survival analysis was estimated according to the Kaplan-Meier method from the date of primary tumor surgery to the time of recurrence of cancer or death from cancer, and the significance of differences between curves was evaluated by the log-rank test. Results were considered statistically significant if the $p$-value was less than 0.05 . The data were analyzed using the SPSS package (version 20.0, SPSS, Inc., Chicago, IL, USA); all $p$-values were two-sided.

\section{Results}

UTUC specimens were obtained from 100 patients with a male-to-female ratio of $1.04: 1.00$. Of these, 62 patients had organ-confined $\mathrm{T}$ stage (T1/T2) cancer and 38 had locally advanced $\mathrm{T}$ stage (T3/T4) cancer. The cancer grade was low in 33 patients and high in 67 patients. Two-thirds of the patients were over 60 years old. Less than half of the patients had abnormal creatinine levels, and only a small proportion $(16 \%)$ needed hemodialysis for end stage renal disease. Activated STAT3 was identified by detection of the phosphorylated form of the protein, p-Ser-STAT3. Figure 1 shows the immunohistochemistry staining of p-Ser-STAT3 in UTUC samples. The patients were stratified into two groups based on low or high p-Ser-STAT3 expression. The clinical parameters and pathological characteristics of the UTUC patients are summarized in Table 1.

Univariate analysis for recurrence-free survival demonstrated that men had higher risk for tumor recurrence $($ Hazard Ratio $[\mathrm{HR}]=2.13,95 \%$ confidence interval $[\mathrm{CI}]=1.06-4.31$ ). Both high grade and late stage were associated with increased risk of tumor recurrence $(\mathrm{HR}=5.09$ and 2.63, respectively). In addition, high p-Ser-STAT3 expression was associated with decreased recurrence-free survival $(\mathrm{HR}=2.4$, $95 \% \mathrm{CI}=1.13-5.08)$. However, following multivariate analysis, only sex and tumor grade were associated with a significantly higher risk of tumor recurrence (Table 2). When patients were stratified based on cancer stage, late stage patients (T3/T4) with high p-Ser-STAT3 protein expression had decreased recurrence-free survival in both univariate $(\mathrm{HR}=4.31$, $\mathrm{p}=0.023)$ and multivariate $(\mathrm{HR}=5.91, \mathrm{p}=0.01)$ analyses compared to patients with low p-Ser-STAT3 expression (Table 3 ).

Table 1. Clinicopathological characteristics of patients with upper tract urothelial carcinoma and association with p-Ser-STAT3 expression.

\begin{tabular}{|c|c|c|c|c|}
\hline \multirow[b]{3}{*}{ Variable } & \multirow[b]{3}{*}{ Patient, no. $(\%)$} & \multicolumn{2}{|c|}{ p-Ser-STAT3 } & \multirow[b]{3}{*}{$p$-Value } \\
\hline & & Low & High & \\
\hline & & $\mathrm{n}(\%)$ & $\mathrm{n}(\%)$ & \\
\hline No. & $100(100.0)$ & $48(48.0)$ & $52(52.0)$ & \\
\hline \multicolumn{5}{|l|}{ Stage } \\
\hline $\mathrm{T} 1 / \mathrm{T} 2$ & $62(62.0)$ & 35 (72.9) & $27(51.9)$ & $0.031^{\mathrm{a}}$ \\
\hline $\mathrm{T} 3 / \mathrm{T} 4$ & $38(38.0)$ & $13(27.1)$ & $25(48.1)$ & \\
\hline \multicolumn{5}{|l|}{ Grade } \\
\hline Low & $33(33.0)$ & $19(39.6)$ & $14(26.9)$ & $0.179 \mathrm{a}$ \\
\hline High & $67(67.0)$ & $29(60.4)$ & $38(73.1)$ & \\
\hline \multicolumn{5}{|l|}{ Gender } \\
\hline Male & $51(51.0)$ & $26(54.2)$ & $25(48.1)$ & $0.543^{\mathrm{a}}$ \\
\hline Female & $49(49.0)$ & $22(45.8)$ & $27(51.9)$ & \\
\hline \multicolumn{5}{|c|}{ Age (years) } \\
\hline$<65$ & $34(34.0)$ & $14(29.2)$ & $20(38.5)$ & $0.327^{a}$ \\
\hline$\geqq 65$ & $66(66.0)$ & $34(70.8)$ & $32(61.5)$ & \\
\hline \multicolumn{5}{|c|}{ Hemodialysis } \\
\hline No & $84(84.0)$ & $37(77.1)$ & $47(90.4)$ & $0.070^{\mathrm{a}}$ \\
\hline Yes & $16(16.0)$ & $11(22.9)$ & $5(9.6)$ & \\
\hline \multicolumn{5}{|c|}{ Creatinine $(\mathrm{mg} / \mathrm{dl})$} \\
\hline$\leqq 1.5$ & $57(57.0)$ & $24(50.0)$ & $33(63.5)$ & $0.174^{\mathrm{a}}$ \\
\hline$>1.5$ & $43(43.0)$ & $24(50.0)$ & $19(36.5)$ & \\
\hline \multicolumn{5}{|c|}{ Recurrence status } \\
\hline No & $68(68.0)$ & $38(79.2)$ & $30(57.7)$ & $0.021^{\mathrm{a}}$ \\
\hline Yes & $32(32.0)$ & $10(20.8)$ & $22(42.3)$ & \\
\hline \multicolumn{5}{|l|}{ Survival } \\
\hline No & $17(17.0)$ & $4(8.3)$ & $13(25.0)$ & $0.034^{\mathrm{b}}$ \\
\hline Yes & $83(83.0)$ & $44(91.7)$ & $39(75.0)$ & \\
\hline
\end{tabular}


Table 2. Univariate and multivariate analysis of recurrence-free survival for patients with upper tract urothelial carcinoma.

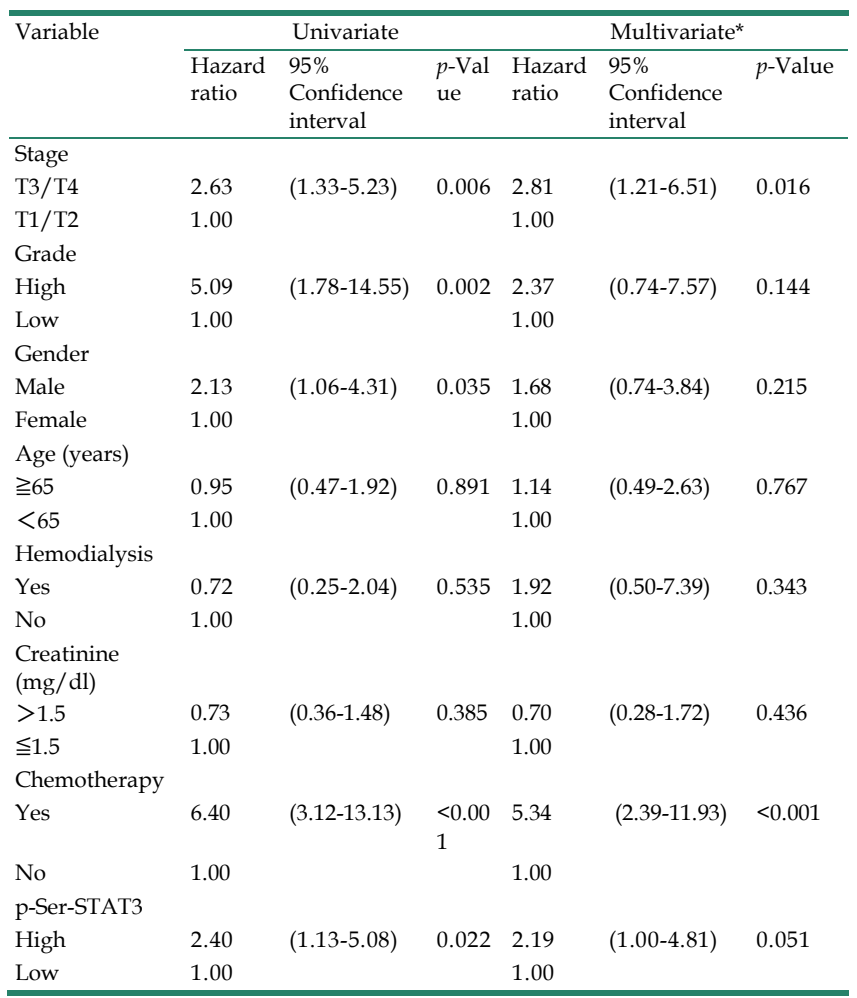

*Multivariate Cox regression model was adjusted for stage, grade, gender, age, hemodialysis, creatinine and chemotherapy.

Univariate analysis also identified cancer stage and p-Ser-STAT3 expression as being associated with cancer-specific survival (Table 4). Late stage and high p-Ser-STAT3 expression were associated with a significant decrease in the cancer-specific survival $(\mathrm{HR}=6.28$ and $\mathrm{HR}=3.32$, respectively $)$. Following multivariate analysis, only cancer stage remained a significant risk factor for cancer-specific survival (HR $=6.03, \mathrm{p}=0.003)$. In patients with late stage $(\mathrm{T} 3 / \mathrm{T} 4)$ UTUC, regardless of cancer grade, high p-Ser-STAT3

(A)

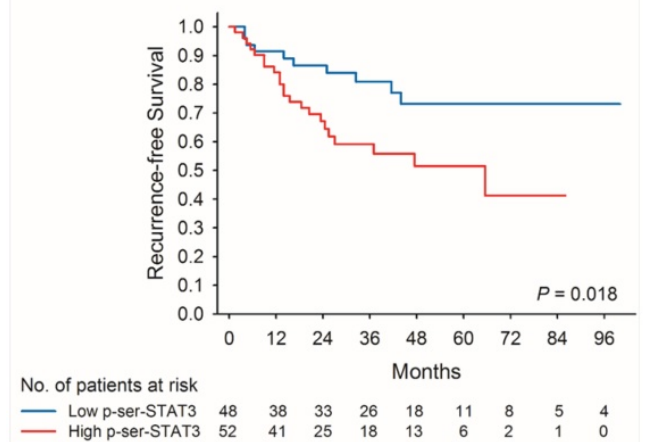

(B)

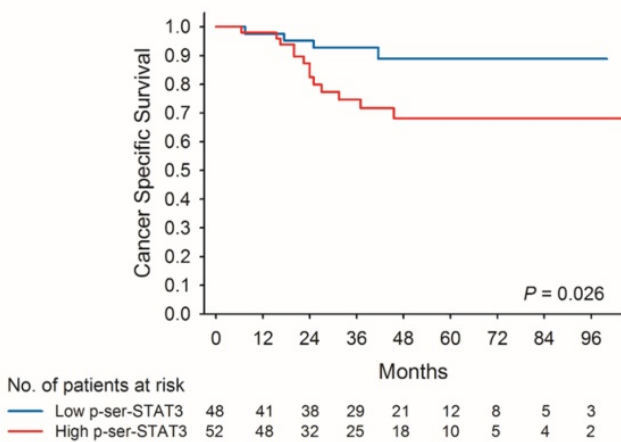

Figure 2. Kaplan-Meier survival curves for recurrence-free $(A)$ and cancer-specific $(B)$ survival of patients with low and high p-Ser-STAT3 expression in all cases of upper tract urothelial carcinoma. expression was the only risk factor associated with cancer-specific survival in both univariate $(\mathrm{HR}=8.13$, $\mathrm{p}=0.045)$ and multivariate analyses $(\mathrm{HR}=8.83, \mathrm{p}=$ 0.039) (Table 5).

Kaplan-Meier survival curves demonstrate that low p-Ser-STAT3 expression was associated with a significantly higher recurrence-free survival $(\mathrm{p}=$ 0.018 ) (Figure 2(A)) and cancer-specific survival ( $\mathrm{p}=$ 0.026 ) (Figure 2(B)). For patients with late stage (T3/T4) disease, high p-Ser-STAT3 expression was associated with a significantly lower recurrence-free survival ( $p=0.013$ ) (Figure 3(B)) and cancer-specific survival $(\mathrm{p}=0.0016)$ (Figure $4(\mathrm{~B}))$. However, this association was not observed in patients with early stage (T1/T2) disease (Figures 3(A) and 4(B)).

\section{Discussion}

As described above, we reported several proteins served as prognostic factors in UTUC patients, including COX2, OPN, HIF-1a, GST, and NFkB. Overexpression of COX2, OPN, HIF-1a and NFKB were associated with poor cancer-specific survival, whereas overexpression of COX2 and HIF-1a could predict shorter recurrence free survival.

In gastric cancer, patients with high levels of STAT3 often experience worse outcomes, with a meta-risk for overall survival (risk ratio, $R R=1.845$ ) [17]. In non-small-cell lung cancer, high STAT3 or phospho-STAT3 expression is also a strong predictor for poor prognosis [18]. High nuclear expression of STAT3 was found to be correlated with poor overall survival $(\mathrm{P}=0.005)$ in diffuse large B-cell lymphoma [19]. Our data indicated that p-Ser-STAT3 expression was also associated with recurrence and survival rates in UTUC patients. Patients with high p-Ser-STAT3 expression had a higher risk for cancer recurrence and a lower cancer-specific survival. When patients were stratified into early and advanced stage groups, there were no significant differences in recurrence-free and cancer-specific survival between low and high p-Ser-STAT3 expression in early stage patients. However, in advanced stage patients, high p-Ser-STAT3 expression was associated with a significantly poorer prognosis, higher cancer recurrence rate, and lower cancerspecific survival. This study provides the first 
evidence suggesting a correlation between p-Ser-STAT3 expression and UTUC.

STAT3 is a transcription factor with important roles in cancer formation and progression. STAT3 is involved in several cellular mechanisms, such as proliferation, inhibition of apoptosis, immune escape, epithelial-mesenchymal transition, invasion, and angiogenesis [20]. In addition, STAT3 is involved in cellular invasion through regulation of matrix metalloproteinases (MMPs) [21] and as first reported by Sano and coworkers. Subsequently, in response to surrounding tumor cell secretions, stromal cells upregulate SDF-1/CXCL12 receptors, resulting in infiltration of endothelial progenitor cells and enhancing the metastatic spread of tumor cells [22]. Under hypoxic conditions, both STAT3 and HIF1-a bind simultaneously to the VEGF promoter, leading to maximum transcriptional activation and angiogenesis. Increased VEGF expression, due to high activation of STAT3, induces faster cancer cell proliferation, and promotes distant metastasis [23]. STAT3, a latent self-signaling transcription factor, has been implicated as the hallmark of tumor invasion and metastasis in a wide variety of human
(A)

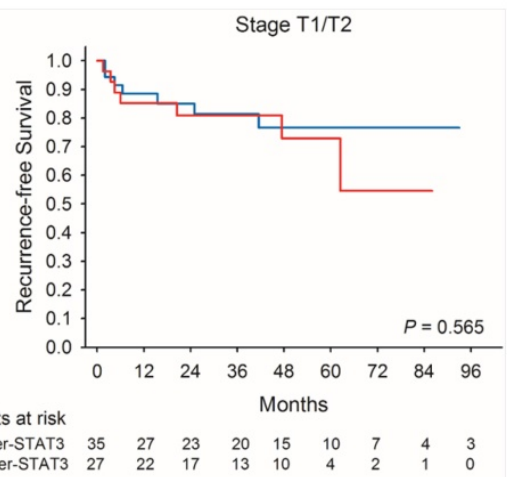

(B)

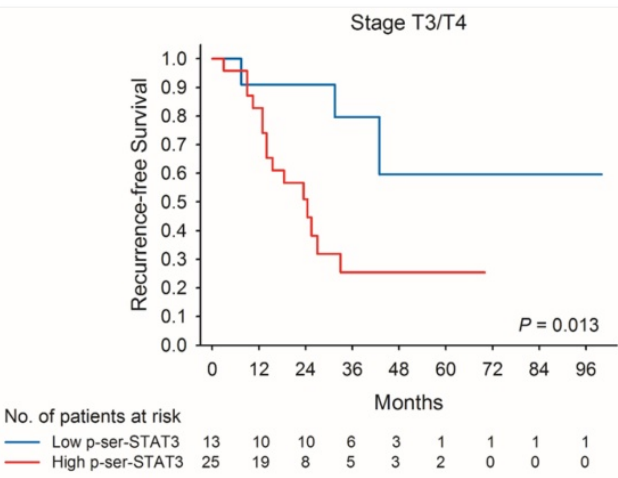

Figure 3. Kaplan-Meier survival curves for recurrence-free survival of patients with low and high p-Ser-STAT3 expression in stage $\mathrm{Tl} / \mathrm{T} 2(\mathrm{~A})$ and stage T3/T4 (B) groups in upper tract urothelial carcinoma.
(A)

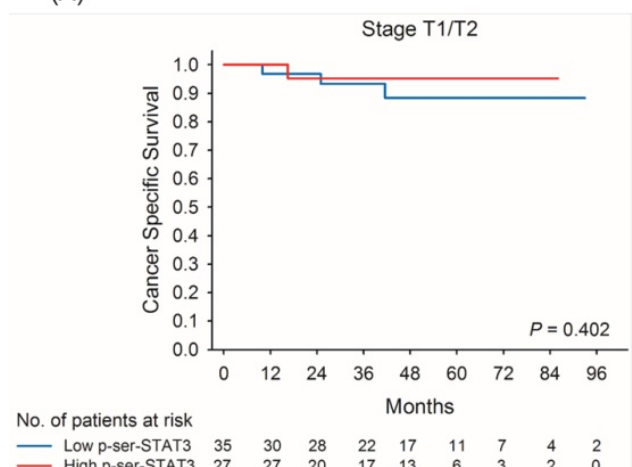

(B)

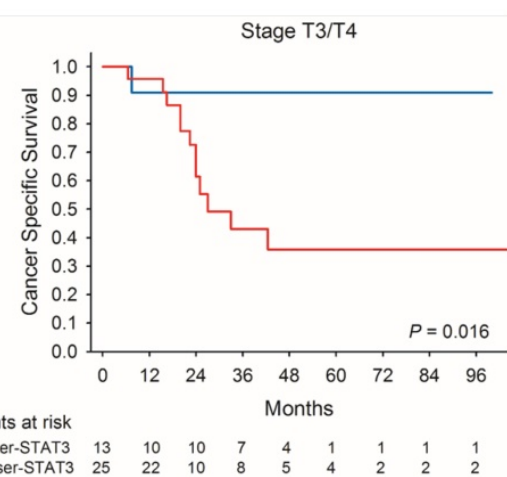

Figure 4. Kaplan-Meier survival curves for cancer-specific survival of patients with low and high p-Ser-STAT3 expression in stage T1/T2 (A) and stage T3/T4 (B) groups in upper tract urothelial carcinoma. malignancies. Activation of STAT3 is achieved by phosphorylation of two sites, tyrosine 705 and serine 727 [24]. Tyrosine 705 phosphorylation is mediated by Jak1, Jak2 and Tyk2, leading to STAT3 homodimerization, nuclear translocation and downstream transcriptional activities. Serine 727 phosphorylation is mediated by ERK1, ERK2, p38, JNK and MAP, which is required for the full activation of STAT3 [25]. Consistent with these studies, we have shown that high p-Ser-STAT3 expression in UTUC tissues significantly correlated with advanced cancer stage and predicting poor prognosis in advanced cancer stage patients. The present investigation revealed that p-Ser-STAT3 overexpression maybe be a useful biomarker to predict disease invasion and metastasis.

On the other hand, inhibiting STAT3 signaling in endothelial cells prohibits cell migration and vessel formation [26]. As metastatic tumor cells enter the blood vessels, they are affected by various nonspecific forces such as mechanical stress, hemodynamic turbulence, loss of adhesion-induced cell death, and cell-mediated cytotoxicity. As a result, very few tumor cells survive and metastasize. STAT3 activation also plays a major role in protecting tumor cells from immune surveillance during their transit through the circulatory system [27]. It is not surprising, therefore, that advanced cancer with lymph-node or organ metastasis requires the specific ability for cell survival with regard to the immune system. The interplay between STAT3 in cancer and immune cells in the tumor microenvironment is very complex and remains elusive. Studies have shown that constitutively active STAT3 signaling recruits immune cells and inhibits their function by increasing suppressive agents [28]. STAT3 mediates bidirectional communication with immune cells and is a potent negative regulator 
of T1 helper cells. In addition, inflammatory cytokines released from tumors, such as IL6 and IL10, are responsible for the partial differentiation of dendritic cells, thereby reducing their antigen presenting ability [29]. By activating the IL-6/STAT3 pathway, the tumor microenvironment promotes tumorigenesis and invasion. Finally, the activity of NK cells also is reduced, thereby protecting circulating tumor cells [30]. The above activities promote the proliferation of cancer cells with a more malignant behavior and contribute to life-threatening disease.

Table 3. Univariate and multivariate analysis of recurrence-free survival for patients with stage T3/T4 in upper tract urothelial carcinoma.

\begin{tabular}{|c|c|c|c|c|c|c|}
\hline \multirow[t]{2}{*}{ Variable } & \multicolumn{3}{|c|}{ Univariate } & \multicolumn{3}{|c|}{ Multivariate $^{*}$} \\
\hline & $\begin{array}{l}\text { Hazard } \\
\text { ratio }\end{array}$ & $\begin{array}{l}95 \% \\
\text { Confidence } \\
\text { interval }\end{array}$ & $p$-Value & $\begin{array}{l}\text { Hazard } \\
\text { ratio }\end{array}$ & $\begin{array}{l}95 \% \\
\text { Confidence } \\
\text { interval }\end{array}$ & $p$-Value \\
\hline \multicolumn{7}{|l|}{ Grade } \\
\hline High & 5.11 & $(0.67-38.82)$ & 0.115 & 5.43 & $(0.64-45.95)$ & 0.120 \\
\hline $\begin{array}{l}\text { Low } \\
\text { Gender }\end{array}$ & 1.00 & \multicolumn{4}{|c|}{ Gender } & \\
\hline Male & 2.48 & $(0.96-6.41)$ & 0.062 & 1.58 & $(0.49-5.09)$ & 0.446 \\
\hline Female & 1.00 & & & 1.00 & & \\
\hline \multicolumn{7}{|c|}{ Age (years) } \\
\hline$\geqq 65$ & 0.55 & $(0.22-1.39)$ & 0.208 & 0.58 & $(0.19-1.74)$ & 0.327 \\
\hline$<65$ & 1.00 & & & 1.00 & & \\
\hline \multicolumn{7}{|c|}{ Hemodialysis } \\
\hline Yes & 0.41 & $(0.06-3.12)$ & 0.392 & 1.95 & $(0.19-20.03)$ & 0.574 \\
\hline No & 1.00 & & & 1.00 & & \\
\hline \multicolumn{7}{|c|}{$\begin{array}{l}\text { Creatinine } \\
(\mathrm{mg} / \mathrm{dl})\end{array}$} \\
\hline$>1.5$ & 0.88 & $(0.33-2.34)$ & 0.795 & 0.80 & $(0.25-2.53)$ & 0.697 \\
\hline$\leqq 1.5$ & 1.00 & & & 1.00 & & \\
\hline \multicolumn{7}{|c|}{ Chemotherapy } \\
\hline Yes & 4.94 & $(1.83-13.38)$ & 0.002 & 3.80 & $(1.08-13.43)$ & 0.038 \\
\hline No & 1.00 & & & 1.00 & & \\
\hline \multicolumn{7}{|c|}{ p-Ser-STAT3 } \\
\hline High & 4.31 & $(1.23-15.11)$ & 0.023 & 6.57 & $(1.71-25.26)$ & 0.006 \\
\hline Low & 1.00 & & & 1.00 & & \\
\hline
\end{tabular}

*Multivariate Cox regression model was adjusted for grade, gender, age, hemodialysis, creatinine and chemotherapy.

Constitutive activation of STAT3 plays a critical role in the initiation, progression, and metastases of cancers. Therefore, inhibition of STAT3 could be a strategy for cancer treatment. Numerous approaches, such as anti-sense oligonucleotide targeting of STAT3, synthetic drugs, small molecules derived from natural sources, and gene therapy techniques are available to achieve this goal [31]. In theory, targeting a single molecular mechanism may be sufficient to be therapeutically effective. However, in practice single-target drugs have had little therapeutic impact and are generally highly ineffective in treating cancer [32]. An approach targeting multiple genes or pathways is likely to be particularly beneficial in cancers because oncogenesis is known to be a multigenic process. Future research focused on the analysis of outcomes and patient prognosis following different targeted therapies in UTUC is needed.

Table 4. Univariate and multivariate analysis of cancer-specific survival for patients with upper tract urothelial carcinoma.

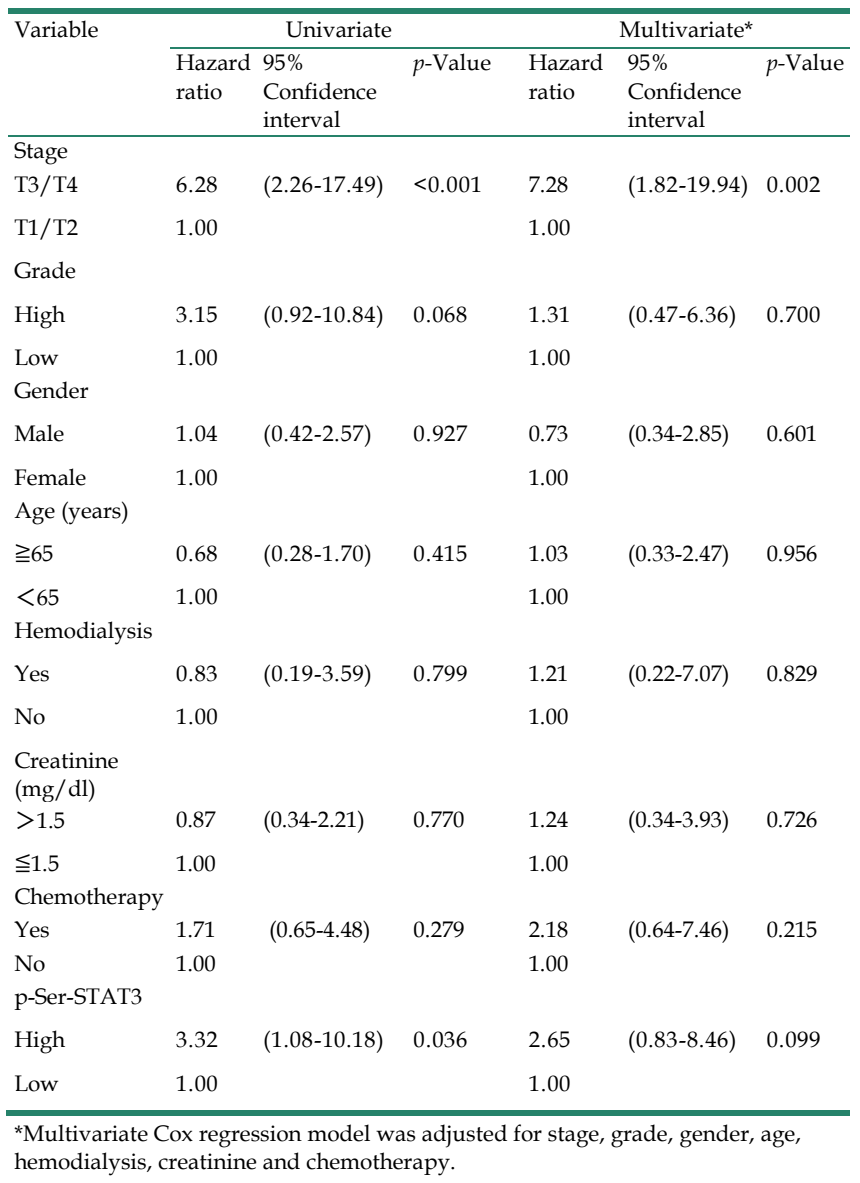

In this study, while stage, grade, and sex were useful predictors of prognosis, high p-Ser-STAT3 expression was a particularly useful marker to predict cancer recurrence and survival. Moreover, high p-Ser-STAT3 expression was the most useful tool for predicting outcome in advanced UTUC. However, there are some limitations to this study. First, this is a retrospective analysis and it neglected the smoking behavior of patients in the subgroup analysis. It has been reported that nicotine can activate STAT3 in bladder cells, thereby affecting cyclin D1 expression and promoting cell proliferation [33]. This confounding factor needs to be addressed in future research. Second, although there was a significant correlation between p-Ser-STAT3 expression and poor outcome, the sample size in this study is small. A multi-institutional study with a larger sample size is needed to validate our conclusions. Finally, easily accessible samples such as blood, urine, or even those 
obtained through minimally invasive ureterorenoscopy tumor biopsies could be analyzed in the future to determine whether similar results are observed regarding the correlation between expression of p-Ser-STAT3 and UTUC patient outcomes. In summary, p-Ser-STAT3 is observed in UTUC and is positively correlated with advanced cancer stage. Moreover, over-expression of p-Ser-STAT3 is predictive of recurrence and survival and may be indicative of the need for an aggressive treatment plan. Furthermore, inhibition of p-Ser-STAT3 may provide a new therapeutic approach for treatment of advanced UTUC.

Table 5. Univariate and multivariate analysis of cancer-specific survival for patients with stage T3/T4 in upper tract urothelial carcinoma.

\begin{tabular}{|c|c|c|c|c|c|c|}
\hline \multirow[t]{2}{*}{ Variable } & \multicolumn{3}{|c|}{ Univariate } & \multicolumn{3}{|c|}{ Multivariate* } \\
\hline & $\begin{array}{l}\text { Hazard } \\
\text { ratio }\end{array}$ & $\begin{array}{l}95 \% \\
\text { Confidence } \\
\text { interval }\end{array}$ & $p$-Value & $\begin{array}{l}\text { Hazard } \\
\text { ratio }\end{array}$ & $\begin{array}{l}95 \% \\
\text { Confidence } \\
\text { interval }\end{array}$ & $p$-Value \\
\hline \multicolumn{7}{|l|}{ Grade } \\
\hline High & 3.02 & $(0.39-23.36)$ & 0.289 & 2.80 & $(0.32-24.57)$ & 0.354 \\
\hline $\begin{array}{l}\text { Low } \\
\text { Gender }\end{array}$ & 1.00 & & & 1.00 & & \\
\hline Male & 1.12 & $(0.38-3.33)$ & 0.842 & 0.92 & $(0.22-3.87)$ & 0.912 \\
\hline \multicolumn{7}{|l|}{ Age (years) } \\
\hline$\geqq 65$ & 0.83 & $(0.28-2.48)$ & 0.737 & 0.98 & $(0.23-4.13)$ & 0.978 \\
\hline$<65$ & 1.00 & & & 1.00 & & \\
\hline \multicolumn{7}{|c|}{ Hemodialysis } \\
\hline Yes & 0.70 & $(0.09-5.42)$ & 0.735 & 1.05 & $(0.09-12.01)$ & 0.968 \\
\hline No & 1.00 & & & 1.00 & & \\
\hline \multicolumn{7}{|l|}{$\begin{array}{l}\text { Creatinine } \\
(\mathrm{mg} / \mathrm{dl})\end{array}$} \\
\hline$>1.5$ & 1.06 & $(0.33-3.46)$ & 0.918 & 1.37 & $(0.26-7.33)$ & 0.716 \\
\hline$\leqq 1.5$ & 1.00 & & & 1.00 & & \\
\hline \multicolumn{7}{|c|}{ Chemotherapy } \\
\hline Yes & 1.42 & $(0.44-4.61)$ & 0.563 & 1.18 & $(0.22-6.42)$ & 0.849 \\
\hline $\begin{array}{l}\text { No } \\
\text { p-Ser-STA }\end{array}$ & 1.00 & & & 1.00 & & \\
\hline High & 8.13 & $(1.05-62.90)$ & 0.045 & 8.93 & $(1.12-71.30)$ & 0.039 \\
\hline Low & 1.00 & & & 1.00 & & \\
\hline
\end{tabular}

*Multivariate Cox regression model was adjusted for grade, gender, age, hemodialysis, creatinine and chemotherapy.

\section{Acknowledgment}

This study was supported by grants from Kaohsiung Medical University "Aim for the Top Universities" (KMU-TP104E31, KMU-TP105G00, KMU-TP105G01 and KMU-TP105G025), The Health and Welfare Surcharge of Tobacco Products, Ministry of Health and Welfare (MOHW106-TDU-B-212 -144007), Ministry of Science and Technology (MOST106-2314-B-037-092), and Kaohsiung Medical University Hospital (KMUH102-2R42 and KMUH1033T11).

\section{Competing Interests}

The authors have declared that no competing interest exists.

\section{References}

1. Roupret M, Babjuk M, Comperat E, Zigeuner R, Sylvester R, Burger M, et al. European guidelines on upper tract urothelial carcinomas: 2013 update. European urology. 2013; 63: 1059-71.

2. Yeh HC, Jan HC, Wu WI, Li CC, Li WM, Ke HL, et al. Concurrent Preoperative Presence of Hydronephrosis and Flank Pain Independently Predicts Worse Outcome of Upper Tract Urothelial Carcinoma. PLoS One. 2015; 10: e0139624.

3. Green DA, Rink M, Xylinas E, Matin SF, Stenzl A, Roupret M, et al. Urothelial carcinoma of the bladder and the upper tract: disparate twins. The Journal of urology. 2013; 189: 1214-21.

4. Ke HL, Tu HP, Lin HH, Chai CY, Chang LL, Li WM, et al. Cyclooxygenase-2 (COX-2) up-regulation is a prognostic marker for poor clinical outcome of upper tract urothelial cancer. Anticancer research. 2012; 32: 4111-6.

5. Ke HL, Chang LL, Yang SF, Lin HH, Li CC, Wu DC, et al. Osteopontin overexpression predicts poor prognosis of upper urinary tract urothelial carcinoma. Urologic oncology. 2011; 29: 703-9.

6. Ke HL, Wei YC, Yang SF, Li CC, Wu DC, Huang CH, et al. Overexpression of hypoxia-inducible factor-1alpha predicts an unfavorable outcome in urothelial carcinoma of the upper urinary tract. International journal of urology : official journal of the Japanese Urological Association. 2008; 15: 200-5

7. Chen $\mathrm{SH}, \mathrm{Wu} \mathrm{WJ}, \mathrm{Tu} \mathrm{HP}, \mathrm{Li} \mathrm{WM}$, Huang $\mathrm{CN}$, Li CC, et al. Glutathione S-transferase expression in upper urinary tract urothelial carcinomas: a Taiwan study. Asian Pacific journal of cancer prevention : APJCP. 2013; 14: 6475-9.

8. Yeh HC, Huang $\mathrm{CH}$, Yang SF, Li CC, Chang LL, Lin HH, et al. Nuclear factor-kappaB activation predicts an unfavourable outcome in human upper urinary tract urothelial carcinoma. BJU international. 2010; 106: 1223-9.

9. Wang X, Crowe PJ, Goldstein D, Yang JL. STAT3 inhibition, a novel approach to enhancing targeted therapy in human cancers (review). Int J Oncol. 2012; 41: 1181-91.

10. Bromberg JF, Wrzeszczynska MH, Devgan G, Zhao Y, Pestell RG, Albanese C, et al. Stat3 as an oncogene. Cell. 1999; 98: 295-303.

11. Darnell JE, Jr. STATs and gene regulation. Science. 1997; 277: 1630-5.

12. Nkansah E, Shah R, Collie GW, Parkinson GN, Palmer J, Rahman KM, et al. Observation of unphosphorylated STAT3 core protein binding to target dsDNA by PEMSA and X-ray crystallography. FEBS Lett. 2013; 587: 833-9.

13. Dolled-Filhart M, Camp RL, Kowalski DP, Smith BL, Rimm DL. Tissue microarray analysis of signal transducers and activators of transcription 3 (Stat3) and phospho-Stat3 (Tyr705) in node-negative breast cancer shows nuclear localization is associated with a better prognosis. Clinical cancer research : an official journal of the American Association for Cancer Research. 2003; 9: 594-600.

14. He G, Karin M. NF-kappaB and STAT3 - key players in liver inflammation and cancer. Cell Res. 2011; 21: 159-68.

15. Masuda M, Suzui M, Yasumatu R, Nakashima T, Kuratomi Y, Azuma K, et al. Constitutive activation of signal transducers and activators of transcription 3 correlates with cyclin D1 overexpression and may provide a novel prognostic marker in head and neck squamous cell carcinoma. Cancer Res. 2002; 62: 3351-5.

16. Huang WT, Yang SF, Wu CC, Chen WT, Huang YC, Su YC, et al. Expression of signal transducer and activator of transcription 3 and suppressor of cytokine signaling 3 in urothelial carcinoma. The Kaohsiung journal of medical sciences. 2009; 25: 640-6.

17. Chen J, Liu X, Jiao H, Peng L, Huo Z, Yang W, et al. Prognostic and clinical significance of STAT3 and MMP9 in patients with gastric cancer: a meta-analysis of a Chinese cohort. Int J Clin Exp Med. 2015; 8: 546-57.

18. Xu YH, Lu S. A meta-analysis of STAT3 and phospho-STAT3 expression and survival of patients with non-small-cell lung cancer. Eur J Surg Oncol. 2014; 40: 311-7.

19. Wu ZL, Song YQ, Shi YF, Zhu J. High nuclear expression of STAT3 is associated with unfavorable prognosis in diffuse large B-cell lymphoma. J Hematol Oncol. 2011; 4: 31.

20. $\mathrm{Yu} \mathrm{H}$, Jove R. The STATs of cancer--new molecular targets come of age. Nat Rev Cancer. 2004; 4: 97-105.

21. Xie T-x, Wei D, Liu M, Gao AC, Ali-Osman F, Sawaya R, et al. Stat3 activation regulates the expression of matrix metalloproteinase-2 and tumor invasion and metastasis. Oncogene. 2004; 23: 3550-60.

22. Nguyen-Jackson H, Panopoulos AD, Zhang H, Li HS, Watowich SS. STAT3 controls the neutrophil migratory response to CXCR2 ligands by direct activation of G-CSF-induced CXCR2 expression and via modulation of CXCR2 signal transduction. Blood. 2010; 115: 3354-63.

23. Oh M-K, Park H-J, Kim N-H, Park S-J, Park I-Y, Kim I-S. Hypoxia-inducible factor-1 1 enhances haptoglobin gene expression by improving binding of STAT3 to the promoter. Journal of Biological Chemistry. 2011; 286: 8857-65.

24. Bishop JL, Thaper D, Zoubeidi A. The Multifaceted Roles of STAT3 Signaling in the Progression of Prostate Cancer. Cancers (Basel). 2014; 6: 829-59. 
25. Yang R, Rincon M. Mitochondrial Stat3, the Need for Design Thinking. Int J Biol Sci. 2016; 12: 532-44.

26. Yahata Y, Shirakata Y, Tokumaru S, Yamasaki K, Sayama K, Hanakawa Y, et al. Nuclear translocation of phosphorylated STAT3 is essential for vascular endothelial growth factor-induced human dermal microvascular endothelial cell migration and tube formation. Journal of Biological Chemistry. 2003; 278: 40026-31.

27. Nguyen DX, Bos PD, Massagué J. Metastasis: from dissemination to organ-specific colonization. Nature Reviews Cancer. 2009; 9: 274-84.

28. Groner B, Lucks P, Borghouts C. The function of Stat3 in tumor cells and their microenvironment. Seminars in cell \& developmental biology: Elsevier; 2008:341-50.

29. Yu H, Pardoll D, Jove R. STATs in cancer inflammation and immunity: a leading role for STAT3. Nat Rev Cancer. 2009; 9: 798-809.

30. Wang T, Niu G, Kortylewski M, Burdelya L, Shain K, Zhang S, et al. Regulation of the innate and adaptive immune responses by Stat-3 signaling in tumor cells. Nature medicine. 2003; 10: 48-54.

31. Siveen KS, Sikka S, Surana R, Dai X, Zhang J, Kumar AP, et al. Targeting the STAT3 signaling pathway in cancer: Role of synthetic and natural inhibitors. Biochimica et Biophysica Acta (BBA)-Reviews on Cancer. 2014; 1845: 136-54.

32. Giordano S, Petrelli A. From single-to multi-target drugs in cancer therapy: when aspecificity becomes an advantage. Current medicinal chemistry. 2008; 15: $422-32$.

33. Chen R-J, Ho Y-S, Guo H-R, Wang Y-J. Rapid activation of Stat 3 and ERK1/2 by nicotine modulates cell proliferation in human bladder cancer cells. Toxicological Sciences. 2008; 104: 283-93. 